\title{
Optimized modelling on lateral separation of twin pontoon-net floating breakwater
}

\author{
A. Fitriadhy ${ }^{1}$, S. F. Abdullah ${ }^{1}$, M. Hairil ${ }^{1}$, M. F. Ahmad ${ }^{1}$, A. Jusoh ${ }^{1}$ \\ ${ }^{1}$ Program of Maritime Technology, School of Ocean Engineering, Universiti Malaysia \\ Terengganu, 21030 Kuala Terengganu, Terengganu, Malaysia \\ *Email: naoe.afit@gmail.com \\ Phone: +6096683856; Fax: +6096683193
}

\begin{abstract}
Since the attribute of wave energy transmission is susceptible to lateral separation (S/D) between twin pontoons of floating breakwater (TPFB), arbitrarily selection of S/D may present problems in the evaluations on the effectiveness of the structure. This paper presents a numerical optimization modelling aimed at obtaining the optimum S/D through Genetic Algorithm (GA) approach. The artificial intelligence is primarily employed to minimize transmission of wave energy coefficients $\left(K_{t}\right)$ whereas maximize energy dissipation coefficient $\left(K_{d}\right)$. To achieve such demand, a numerical simulation implementing a MATLAB code as an interface between the Genetic Algorithm and a CFD program is applied. Several parameters for the effects of various wavelengths and ratios of S/D including a set of criteria have been considered in the simulation, where the optimum solution is chosen from various populations. The results demonstrated that the current GA analysis is efficient that can search a global trade-off between $K_{t}$ and $K_{d}$ to determine an optimum S/D ratio. For S/D equal to 2.72, $K_{t}$ minimized to less than 0.3 as compared to existing model $\left(K_{t}>0.5\right)$ while $K_{d}$ maximized to greater than 0.95 resulting to optimum hydrodynamic effects of TPFB. Hence, the optimization algorithm can serve as a useful engineering tool for a conceptual design to determine an optimum S/D for twin pontoon floating breakwater.
\end{abstract}

Keywords: Genetic algorithm; optimization; twin pontoon floating breakwater; lateral separation; hydrodynamic coefficients; computational fluid dynamics (CFD).

\section{INTRODUCTION}

Floating breakwaters increasingly become an alternative and reliable coastal protection as it is cheaper in production cost as compared to conventional bottom-fixed breakwaters. Floating breakwater system can flexibly oriented on location while adapt with sea level rise and fall that would be a best decision in order to control the sedimentation that threatens the shore due to erosion [1]. Their utilization is further enhanced in circumstances for water circulation and esthetic considerations [2] apart from multiple uses as walkways, docks, marine culture etc. Hence, floating breakwaters are paid growing attention among researchers in the area of coastal and offshore research.

Pontoons can be regarded as the most common and simple types of floating breakwater which have gained much interests in the literature. Single pontoon type of floating breakwater 
has been studied by many researchers [3-6], each of these investigations had devoted to the efficiency of the structure in respects to hydrodynamic coefficients, motion response and mooring force. Many recent researches have been for twin pontoons of floating breakwater (TPFB) such as cage floating breakwater [7, 8] and cylindrical floating breakwater [9-11]. Instead of adding total weight, the inertia of TPFB can simply increases by adjusting its lateral separation, S/D (ratio of distance between two pontoons over its width). Since S/D is purely frequency dependence, optimizing this parameter importantly enhances stability and wave attenuation of the structure.

For examples, Brebner and Ofoya [12] and Williams and Abul Azm [13] applied W/L (ratio of breakwater width to incident wavelength) greater than 0.60 for TPFB to obtain $K_{t}$ less than 0.50. Similarly, Syed and Mani [14] studied a multiple interconnected pontoon with S/D equal to 0.50 to obtain the same wave attenuation. Recently, a TPFB with mesh cage and suspended balls had been investigated by some authors in moored [9] and pile restrained [15] conditions. In their study, the new type of floating breakwater exhibits a better performance in long waves, however the variable trends of $K_{t}$ are mostly higher than 0.5 . In fact, the basis for determining the optimum S/D of TPFB is still unclear and insufficiently available in literature. This may result in a difficulty to evaluate the hydrodynamic performance of such structure largely due to arbitrarily selection of this principal dimension.

One of the popular and reliable approaches towards structure shape optimization is using optimization algorithms. Despite many types of optimization methods had been appeared over years (e.g. simulated annealing, artificial neural network, particle swarm optimization etc.), genetic algorithm (GA) remains the most robust search technique for multiobjective optimization problems since it uses evolutionary strategies namely cross over and mutation which firmly improve the initial solutions from local to global optimal solutions [16]. Moreover, the resulting solution is often optimum or near to optimum over an appropriate time [17]. Therefore, in this study, the optimization on S/D of TPFB is performed using multi-objective genetic algorithm preceding by a parametric study for various S/D ratios and wavelengths by means of CFD approach. The optimization algorithm is primarily applied to minimize and maximize the wave transmission and energy dissipation respectively.

\section{GENETIC ALGORITHM}

Inspired from biological evolution, Genetic Algorithms (GAs) are a population-based optimization technique based on the Darwinian theory of survival of the fittest [18]. In this study, the real coded of Elitist non-dominated sort GA (NSGA-II) is deliberately used. Basically, the NSGA-II [19] is one of the popularly used evolutionary multi-objective optimization (EMO) procedures that attempt to find multiple Pareto-optimal solutions in a multi-objective optimization problem. It utilizes an elitist principle and explicit diversity preserving mechanism, as well emphasizes non-dominated solutions.

The procedure of GA process and techniques is presented as in the Figure 1. The particular breakwater to be analyzed basically consists of two pontoons with fish net attached underneath. To begin, the model validation is firstly carried out for the floating breakwater under various wave heights and wavelengths using computational fluid dynamic (CFD). The transmission coefficient is properly estimated and compared accordingly with experimental data reported in the literature. After a reasonable agreement is achieved, the CFD solver is 
further used for the analysis of wave interaction with the structure at various wavelengths and lateral separation ratios, S/D in which the equations of wave transformations are correspondingly derived. The pertaining equations is then feed into the objective functions to be evaluated by genetic algorithm.

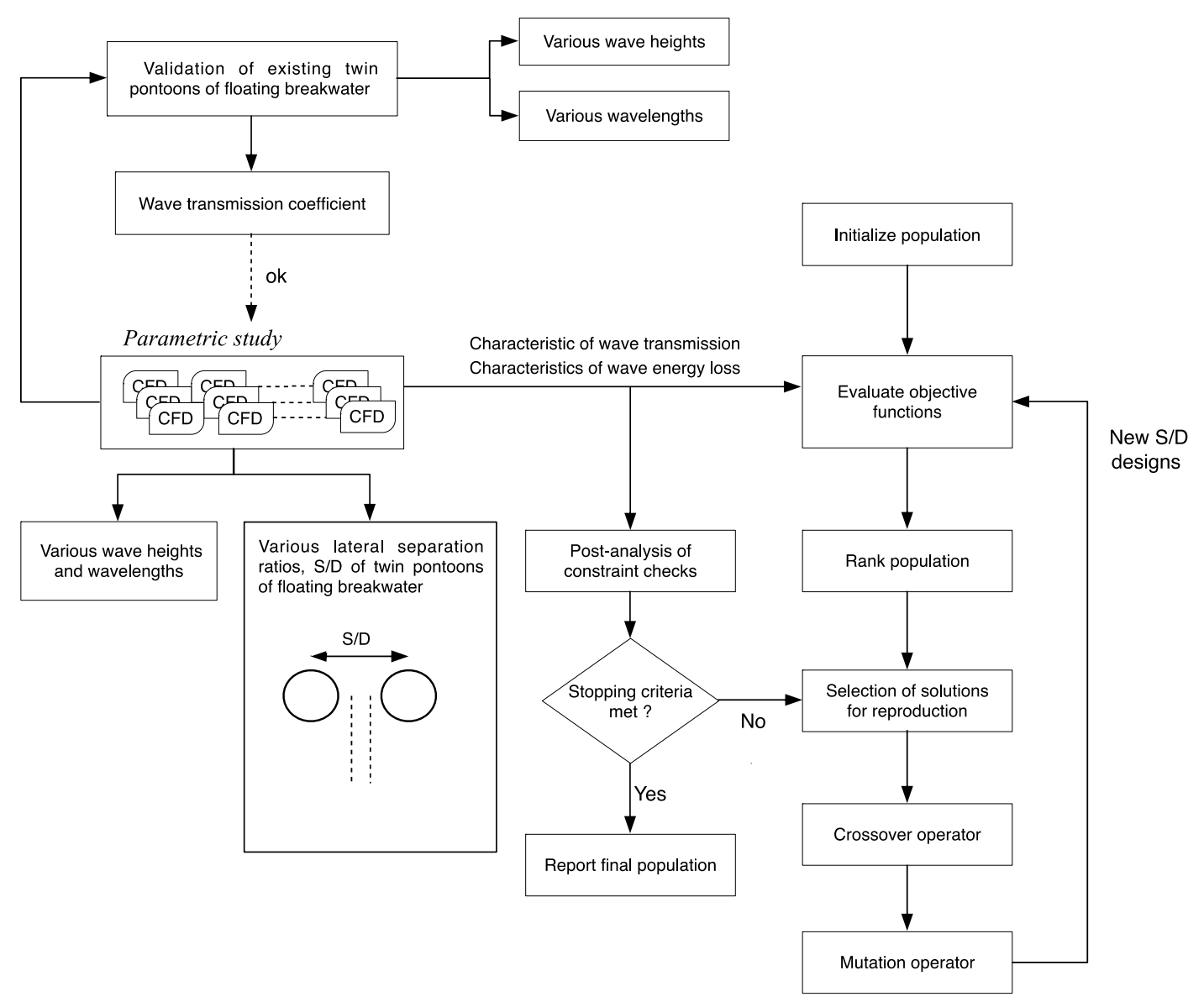

Figure 1. Flowchart of the methodology and GA process.

At any generation, the offspring population is created by using the parent of initial population and the usual genetic operators. Thereafter, the parent and offspring populations are firmly combined together to form a new chromosomes with different non-domination classes and diversities (crowding distance) [19]. Here, the non-dominated solutions comprising of numerous S/D chromosomes are created and again reevaluated with respect to defining constraints. The optimum solution is finally chosen from a global optimal set of nondominated solutions with various populations over multiple steps 2-4 and generations in which some convergence criterion is met. During optimization, tuning on important GA parameters such as crossover rate and mutation rate are necessary in order to avoid current search traps at local optimal solutions. For the implementation details of the genetic operators the authors refer to $[20,21]$. 


\section{HYDRODYNAMICS PERFORMANCE ANALYSIS}

For the purpose of hydrodynamics analysis of twin pontoon-net floating breakwater, the Flow3D solution is applied to describe the fluid and structure dynamics. The CFD flow solver on Flow3D version 10.1 is based on the incompressible unsteady RANS equations in which the solver applies the Volume of Fluid (VOF) to track the free surface elevation. The interface between fluid and solid boundaries is simulated with the fractional area volume obstacle representation favor method [22]. This method computes open area and volume in each cell to define the area that is occupied by obstacle [23]. Flow3D employs the meshing method FAVOR ${ }^{\mathrm{TM}}$ that dramatically improves problem setup by embedding the geometry directly into the mesh, allowing for rapid parametric adjustments without the labor-intensive remeshing required by other CFD software.

\section{Continuity and Momentum Equation}

The continuity and momentum equations for a moving object and the relative transport equation for VOF function are:

$$
\begin{aligned}
\frac{V_{f}}{\rho} \frac{\partial \rho}{\partial t}+\frac{1}{\rho} \nabla \cdot\left(\rho \vec{u} A_{f}\right) & =-\frac{\partial V_{f}}{\partial t} \\
\frac{\partial \vec{u}}{\partial t}+\frac{1}{V_{f}}\left(\vec{u} A_{f} \cdot \nabla \vec{u}\right) & =-\frac{1}{\rho}\left[\nabla_{\rho}+\nabla \cdot\left(\tau A_{f}\right)\right]+\vec{G} \\
\frac{\partial F}{\partial t}+\frac{1}{V_{f}} \nabla \cdot\left(F \vec{u} A_{f}\right) & =-\frac{F}{V_{f}} \frac{\partial V_{f}}{\partial t}
\end{aligned}
$$

where $\rho$ is the density of the fluid, $\vec{u}$ is the fluid velocity, $V_{f}$ is the volume fraction, $A_{f}$ is the area fraction, $p$ is the pressure, $\tau$ is the viscous stress tensor, $G$ denotes gravity and $F$ is the fluid fraction.

In the case of coupled GMO's motion, Eqs. (1) and (2) are solved at each time step and the location of all moving objects is recorded and the area and volume fractions updated using the FAVOR technique. Eq. (3) are solved with the source term $\left(-\frac{\partial V_{f}}{\partial t}\right)$ on the righthand side which is computed as:

$$
-\frac{\partial V_{f}}{\partial t}=\vec{U}_{o b j} \cdot \vec{n} S_{o b j} / V_{c e l l}
$$

where $S_{o b j}$ is the surface area, $\vec{n}$ surface normal vector, $\vec{U}_{o b j}$ is the velocity of the moving object at a mesh cell and $V_{\text {cell }}$ is the total volume of the cell [23].

\section{Turbulence Model}

The RNG turbulence model was used for the simulation of the exchange flow between open water and floating object since it accounts for low Reynolds number effects [24]. Applying the double averaging strategy to the transport equations for turbulent kinetic energy (TKE) and its dissipation rate produces the turbulence model for the flow. The resulting equations are:

$$
\begin{gathered}
\frac{\delta \mathrm{k}}{\delta \mathrm{t}}+\mathrm{U}_{\mathrm{j}} \frac{\delta \mathrm{k}}{\delta \mathrm{x}_{\mathrm{j}}}=\frac{\delta}{\delta \mathrm{x}_{\mathrm{j}}}\left[\left(\mathrm{v}+\frac{\mathrm{v}_{\mathrm{t}}}{\sigma_{\mathrm{k}}}\right) \frac{\delta \mathrm{k}}{\delta \mathrm{x}_{\mathrm{j}}}\right]+\mathrm{P}_{\mathrm{k}}+\mathrm{B}_{\mathrm{k}}+\mathrm{W}_{\mathrm{k}}-\varepsilon \\
\frac{\delta \varepsilon}{\delta \mathrm{t}}+\mathrm{U}_{\mathrm{j}} \frac{\delta \varepsilon}{\delta \mathrm{x}_{\mathrm{j}}}=\frac{\delta}{\delta \mathrm{x}_{\mathrm{j}}}\left[\left(\mathrm{v}+\frac{\mathrm{v}_{\mathrm{t}}}{\sigma_{\varepsilon}}\right) \frac{\delta \varepsilon}{\delta \mathrm{x}_{\mathrm{j}}}\right]+\mathrm{C}_{1 \varepsilon} \frac{\varepsilon}{\mathrm{k}}\left(\mathrm{P}_{\mathrm{k}}+\mathrm{B}_{\mathrm{k}}\right)\left(1+\mathrm{C}_{3 \varepsilon} \mathrm{R}_{\mathrm{f}}\right)+\mathrm{W}_{\varepsilon}-\mathrm{C}_{2 \varepsilon}^{*} \frac{\varepsilon^{2}}{\mathrm{k}}
\end{gathered}
$$




$$
\begin{aligned}
& \mathrm{P}_{\mathrm{k}}=\mathrm{v}_{\mathrm{t}} \mathrm{S}^{2}=\mathrm{v}_{\mathrm{t}}\left(\frac{\delta \mathrm{U}_{\mathrm{i}}}{\delta \mathrm{x}_{\mathrm{j}}}+\frac{\delta \mathrm{U}_{\mathrm{j}}}{\delta \mathrm{x}_{\mathrm{i}}}\right) \frac{\delta \mathrm{U}_{\mathrm{i}}}{\delta \mathrm{x}_{\mathrm{j}}} \\
& \mathrm{B}_{\mathrm{k}}=\beta \mathrm{g}_{\mathrm{i}} \frac{\mathrm{v}_{\mathrm{t}}}{\sigma_{\mathrm{s}}} \frac{\delta \mathrm{s}}{\delta \mathrm{x}_{\mathrm{i}}}
\end{aligned}
$$

where $\mathrm{P}_{\mathrm{k}}$ is the shear production term of TKE, $S=\sqrt{2 S_{i j} S_{j i}}$ is the modulus of the mean rate of strain tensor and $S_{i j}=\frac{1}{2}\left(\frac{\delta U_{i}}{\delta x_{j}}+\frac{\delta U_{j}}{\delta x_{i}}\right), B_{k}$ is the buoyant production term of TKE, $W_{k}$ is the wake production term of TKE, $W_{\varepsilon}$ is the wake production term in $\varepsilon, \sigma_{k}$ and $\sigma_{\varepsilon}$ are the turbulent Prandtl numbers for $k$ and $\varepsilon$, and $C_{i \varepsilon}, C_{3 \varepsilon}$ and $C_{2 \varepsilon}^{*}$ are model coefficients.

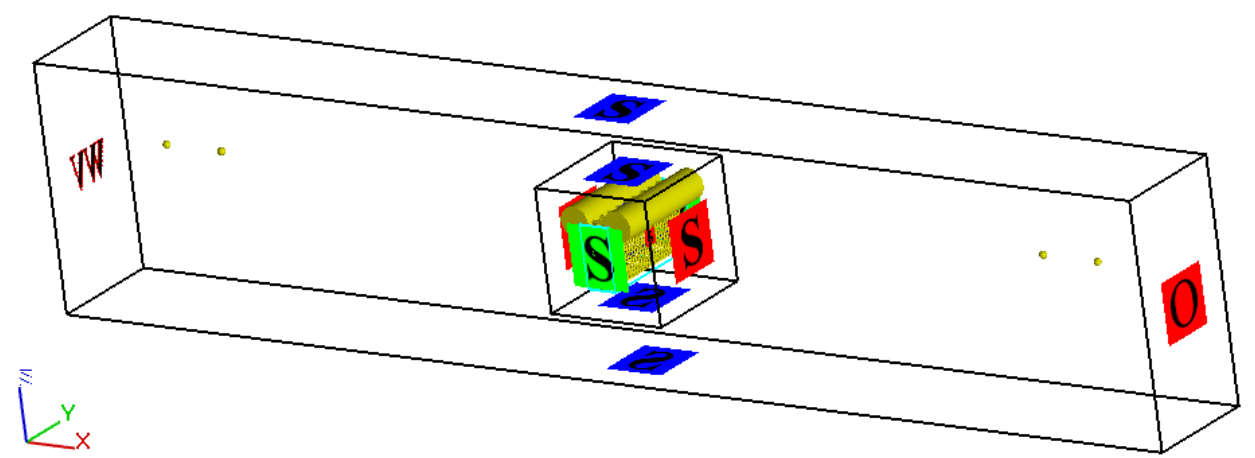

Figure 2. Boundary condition in the computational domain.

\section{Computational Domain}

In the innovative procedure, the breakwater model is properly developed in CAD and then integrated with CFD techniques. The motion response of the structure is described in heave degree of freedom (DOF). Using appropriate set-up, the effective domains for this CFD simulation is depicted as in Figure 2 and Table 1.

Table 1. Computational domain and boundary setting conditions.

\begin{tabular}{lccl}
\hline Description & $\begin{array}{c}\text { Distance } \\
\text { with respect } \\
\text { to origin } \\
\text { point }\end{array}$ & Type & Condition \\
\hline $\boldsymbol{X}_{\text {min }}$ & $12.0 d$ & Wave & Far field \\
$\boldsymbol{X}_{\text {max }}$ & $12.0 d$ & Outflow & Far field \\
$\boldsymbol{Z}_{\text {min }}$ & $1.00 d$ & Symmetry & Far field \\
$\boldsymbol{Z}_{\text {max }}$ & $0.60 d$ & Symmetry & Far field \\
$\boldsymbol{Y}_{\text {min }}$ & $0.40 d$ & Symmetry & Far field \\
$\boldsymbol{Y}_{\max }$ & $0.40 d$ & Symmetry & Far field \\
\hline
\end{tabular}

Referring to Table 1, the wave boundary condition is assigned for the upstream while the outflow boundary condition for the downstream and symmetrical type for all other open boundaries is to minimize the effects of friction loss and surface tension. 


\section{Meshing Generation}

The suitable mesh element for the domain discretization is important in order to maintain numerical accuracy and steadiness in the computational results regardless of longer CPU time. Based on a mesh independent study showed in Table 2, the total of 3 million cells of case D was adopted for the simulations since further increments of total cells was unnecessary on account of its insignificant influence into the computational results.

Table 2. Mesh independent study.

\begin{tabular}{lccc}
\hline Case & Mesh type & $\begin{array}{c}\text { Total number } \\
\text { of cell meshing }\end{array}$ & $\boldsymbol{K}_{\boldsymbol{t}}$ \\
\hline A & Rectangular & 196608 & 0.796 \\
B & Rectangular & 384000 & 0.565 \\
C & Rectangular & 1572864 & 0.424 \\
D & Rectangular & 3072000 & 0.415 \\
E & Rectangular & 6000000 & 0.420 \\
\hline
\end{tabular}

\section{Regular Wave Theory}

In the simulation, the linear wave theories have been used in the solution corresponding to wave height, $H=0.2 \mathrm{~m}$ and wave period, $T=1.7 \mathrm{~s}$. The regular wave equation for the free surface elevation $\eta(x, t)$, the velocity potential $\varphi(x, z, t)$, and velocity components in $x$ and $z$ directions $u(x, z, t)$ and $\mathfrak{w}(x, z, t)$ are rewritten as [23],

$$
\begin{aligned}
& \eta=A \cos (k x-\omega t+\phi) \\
& \varphi(x, z, t)=x U+\frac{A \omega \cosh [k(z+h)] \sin (k x-\omega t+\phi)}{k \sinh k h} \\
& u(x, z, t)=U+\frac{A \omega \cosh [k(z+h)] \cos (k x-\omega t+\phi)}{\sinh k h} \\
& \mathfrak{w}(x, z, t)=\frac{A \omega \sinh [k(z+h)] \sin (k x-\omega t+\phi)}{\sinh k h}
\end{aligned}
$$

where $\omega$ is the angular frequency, $k$ is the wave number and $\phi$ is the phase shift angle. The dispersion equation in terms of wave speed $c=\omega / k$ is given by:

$$
(c-U)^{2}=\frac{g}{k} \tanh k h
$$

For further analysis scheme of hydraulic data, the authors used two-probes method introduced by Goda and Suzuki (1977) [25] for separation between reflected waves $\left(A_{r}\right)$ and incident waves $\left(A_{i}\right)$ and also for transmitted wave $\left(A_{t}\right)$. The dimensionless parameters of transmission $\left(K_{t}\right)$, reflection $\left(K_{r}\right)$ and energy dissipation $\left(K_{d}\right)$ coefficients that can be estimated as: 


$$
\begin{gathered}
K_{t}=A_{t} / A_{i} \\
K_{r}=A_{r} / A_{i} \\
K_{d}=\sqrt{1-K_{t}{ }^{2}-K_{r}{ }^{2}}
\end{gathered}
$$

\section{SIMULATION CONDITION}

\section{Model}

Figure 3 shows the detail configuration of twin pontoon-net floating breakwater. In the simulation, the particular model of floating breakwater was constructed based on a dimensional and geometrical similarity of 1:20. The virtual model is basically consisting of two main cylinders, $0.2 \mathrm{~m}$ (diameter) connected along their perpendicular directions by nine close-spaced mini-cylinders (connectors), $0.02 \mathrm{~m}$ (diameter) thus jointly together forming superstructure of the breakwater system. Apart from the main body, the flexible structure of net cage of $0.1 \mathrm{~m}$ wide and 0.4 high is designed hanging below the superstructure specifically to damp wave energy at a greater depth (see Figure 3). In addition, a curtain was embedded between two floating cylinders should specifically to facilitate better wave breaking before the curtain especially in high amplitude waves and it is also intended to reduce the pressure and water particle velocities inside the gap since the flow parameters can significantly affect wave transmission of the floating breakwater. Table 3 presents the geometrical and structural characteristics of the structure used in the CFD simulation.

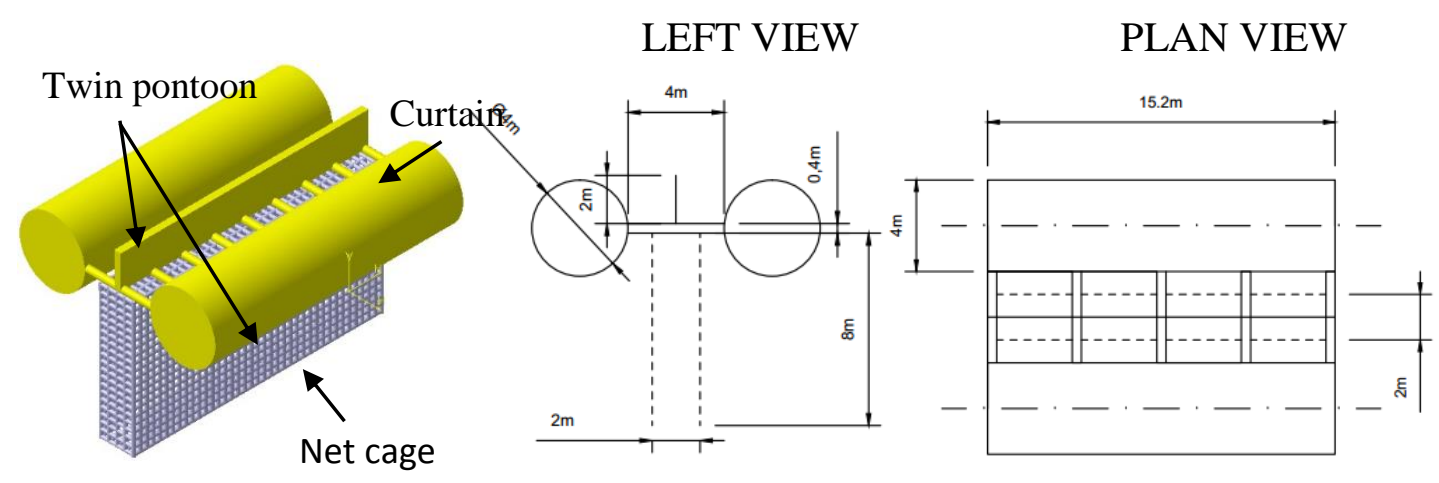

Figure 3. Geometric configuration of twin pontoon-net floating breakwater. 
Table 3. Geometrical and structural characteristics of twin pontoon-net floating breakwater model.

\begin{tabular}{lccc}
\hline Characteristics & & Value \\
\cline { 1 - 3 } Descriptions & $\boldsymbol{l}$ & Unit & \\
\hline Length & $\boldsymbol{W}$ & $\mathrm{m}$ & 0.7600 \\
Width & $\boldsymbol{D}$ & $\mathrm{m}$ & 0.5000 \\
Height & $\boldsymbol{d}$ & $\mathrm{m}$ & 0.5100 \\
Draft & $\boldsymbol{m}$ & $\mathrm{kg}$ & 0.4100 \\
Mass & $\boldsymbol{I}_{\boldsymbol{x} \boldsymbol{x}}$ & $\mathrm{kg} * \mathrm{~m}^{2}$ & 33.418 \\
Roll inertia & $\boldsymbol{I}_{\boldsymbol{y} \boldsymbol{y}}$ & $\mathrm{kg} * \mathrm{~m}^{2}$ & 2.6496 \\
Pitch inertia & $\boldsymbol{I}_{\boldsymbol{z z}}$ & $\mathrm{kg} * \mathrm{~m}^{2}$ & 1.6056 \\
Yaw inertia & $\boldsymbol{C}_{\boldsymbol{g}}$ & $\mathrm{m}$ & 2.3246 \\
Initial mass center & & & 0.0250 \\
location & & & \\
\hline
\end{tabular}

\section{PARAMETRIC STUDY}

\section{Study Parameter}

Differ from traditional breakwaters, the wave attenuating mechanism of floating breakwater is perceived as either to reflect, dissipate or transmit the wave to leeside of the structure or the combinations of each. Hence, the hydrodynamics performance of the structure can be evaluated via determination of the wave transformation characteristics consist of transmission, reflection and energy dissipation. In further, the effect of lateral separation, S/D of twin pontoon-net floating breakwater on the aforementioned wave coefficients is depicted in Figure 4. Meanwhile, Table 4 summarizes various tested S/D parameters used in the simulation. Accordingly, a GA optimization model of S/D will be developed on the basis of the results of present parametric study.

Table 4. Numerical test condition.

\begin{tabular}{cc}
\hline Diameter, D $(\mathbf{m})$ & S/D \\
\hline & 2.000 \\
2.125 \\
2.250 \\
2.375 \\
0.2 & 2.500 \\
& 2.625 \\
& 2.750 \\
& 2.875 \\
& 3.000 \\
& 3.125 \\
\hline
\end{tabular}




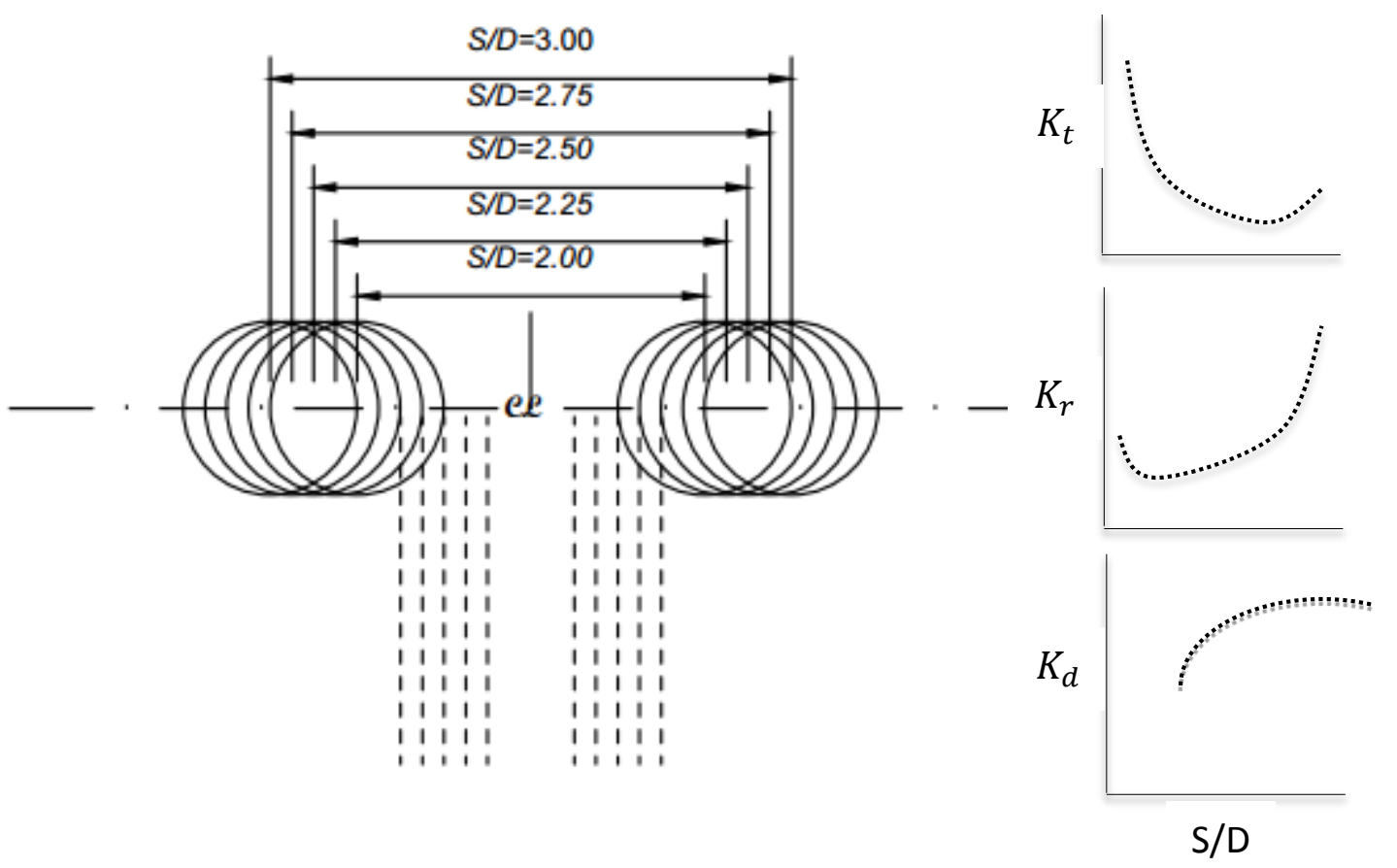

Figure 4. Effect of S/D of twin pontoon-net floating breakwater on transmission $K_{t}$, reflection $K_{r}$ and energy dissipation $K_{d}$ coefficients.

\section{MATHEMATICAL FORMULATION}

Since objectives can be either minimized or maximized, the present optimization problem of hydrodynamics performance of twin pontoon-net floating breakwater is formulated in the following form:

Find $s_{x}$ which:

Subject to:

$$
\begin{array}{ll}
\text { Minimize } & f_{1}\left(s_{x}\right)=K_{t} \\
\text { Maximize } & f_{2}\left(s_{x}\right)=K_{d}
\end{array}
$$

$$
\begin{array}{lc}
x_{i}^{(L)} \leq x_{i} \leq x_{i}^{(U)} & i=1,2, \ldots, I \\
g_{j}(x) \leq 0.3, & j=1,2, \ldots, J \\
b_{l}(x) \geq 0.8, & l=1,2, \ldots, L \\
h_{k}(x)=1.0, & k=1,2, \ldots, K
\end{array}
$$

where, $f_{1}\left(s_{x}\right)$ and $f_{2}\left(s_{x}\right)$ are the objective functions, which can be defined in this research, in correspondence to the problem as transmission and energy dissipation coefficients respectively. In Eq. (19), the lower, $L$ and upper, $U$ variable bounds for the decision variable, $s_{x}$ in search space is defined, which refer to lateral separation between twin pontoons of floating breakwater.

Among detail criteria of present optimization problems are formulated as in Eq. (20) and Eq. (21) with corresponding to the requirement for wave attenuation and dissipation from 
the structure respectively. For example, the floating breakwater should not transmit higher than $30 \%$ of incident waves at maximum while it should dissipate minimally more than $80 \%$ of wave energy. In order to ensure a realistic search of GA, additional constraint of the problem is used that all objective results should satisfy energy equilibrium of Eq. (22). In this technique, the authors intrinsically embed the reflection characteristics of the floating breakwater into above formulation.

In each generation of the GA process, the solution vectors $s_{x}$ in current population and off springs are sorted and selected based on the rank of their non-domination and crowding distance. Then, the solutions with best rank and diversity are chosen for reproduction, and those with lowest rank and crowding distance are gradually eliminated.

\section{RESULTS AND DISCUSSION}

Figures 5 to 13 show the CFD and optimization results have been successfully computed in this study. The pertaining discussions are appropriately presented in the following subsections.

\section{Validation of Floating Breakwater Model}

The wave damping performance of floating breakwater under sea wave force is estimated by its wave transmission coefficient, $K_{t}$. The validation purpose for the present study has been based on experimental study of similar twin pontoons called cylindrical floating breakwater (CFB) by ji et. al [9]. Figure 5 shows the change in transmission coefficient, $K_{t}$ with various wave periods for CFD method and experimental model. The results generally show a qualitative and quantitative agreement is observed when comparing the results of $K_{t}$ for CFD with the experiment model test. Meanwhile, the discrepancies between two results are approximately from 0.554 to $13.726 \%$.
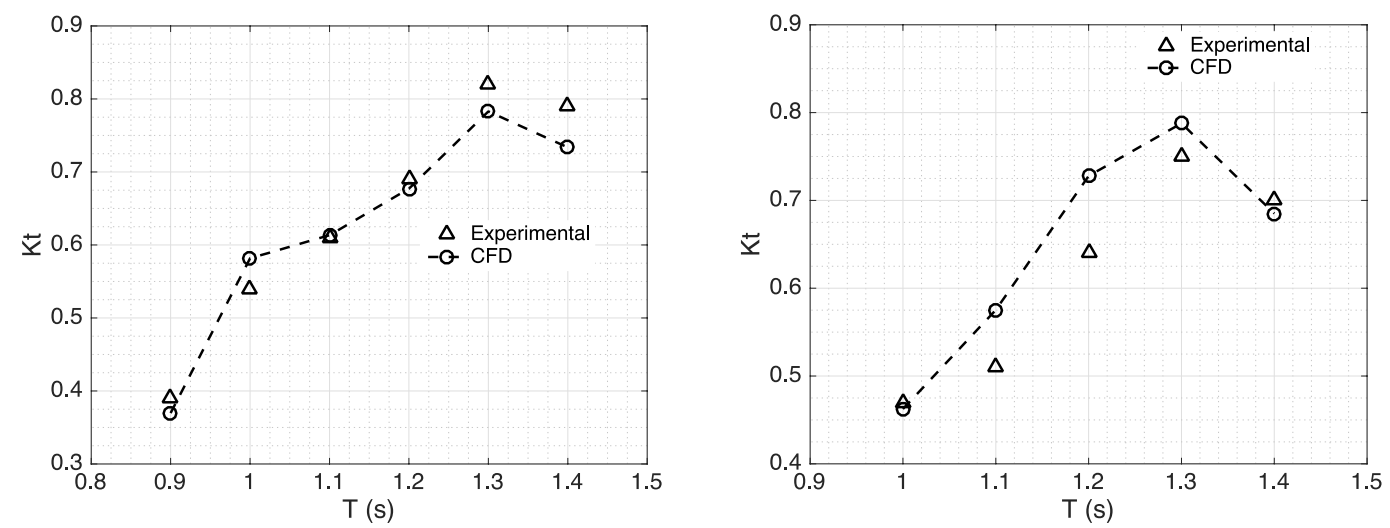

Figure 5. Transmission coefficients for (a) $H=0.15 \mathrm{~m}$, (b) $H=0.2 \mathrm{~m}$.

Further, it is interesting to note that the present model is being able to validate the wave attenuating effects of CFB especially for longer waves $(T=1.4 \mathrm{~s})$ similar to experimental one. As depicted in Figure 5 (a) and (b), $K_{t}$ climbs with increasing wavelength up to $1.3 \mathrm{~s}$ wave period at approximately 0.78 and 0.79 wave transmission for respective 0.15 and 0.2 
m wave height. Beyond this, the $K_{t}$ declines with marked effect for the figure of $\mathrm{H}=0.2 \mathrm{~m}$ at $K_{t}$ around 0.69 . This is also supported by the reduction of flow parameter observed in CFD simulation as shown in Figure 6.
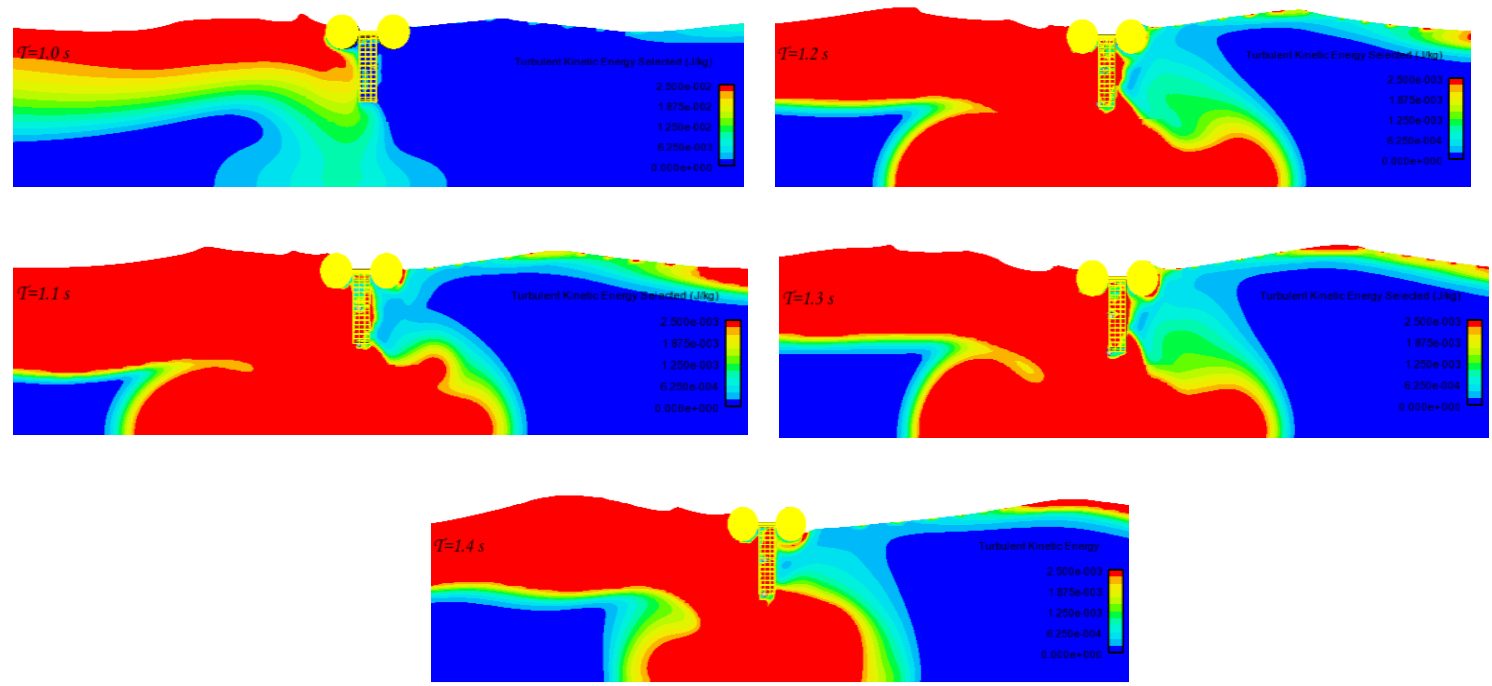

Figure 6. Wave absorbing effects of CFB in wave energy pattern for a) $\mathrm{H}=0.15 \mathrm{~m}, \mathrm{~b}$ ) $\mathrm{H}=$ $0.2 \mathrm{~m}$.

In Figure 6, it is conveniently to explain that the wave energy before and after the floating breakwater increases as the wave period increases which can be traced by the red-green scale color. Proportionally, the energy difference between the two referred sides also becomes closer up to $\mathrm{T}=1.3 \mathrm{~s}$ indicating increased transmission by CFB. However, for $\mathrm{T}=1.4 \mathrm{~s}$, the difference is slightly released while exhibiting more spreading patterns should the reason for the increased wave attenuating effect of CFB beyond $1.3 \mathrm{~s}$ wave period. One of the reasons is due to increased viscous and drag damping from increasing flow rate of water across the net cage of CFB that is proportional to the amount of pressure and energy drop.

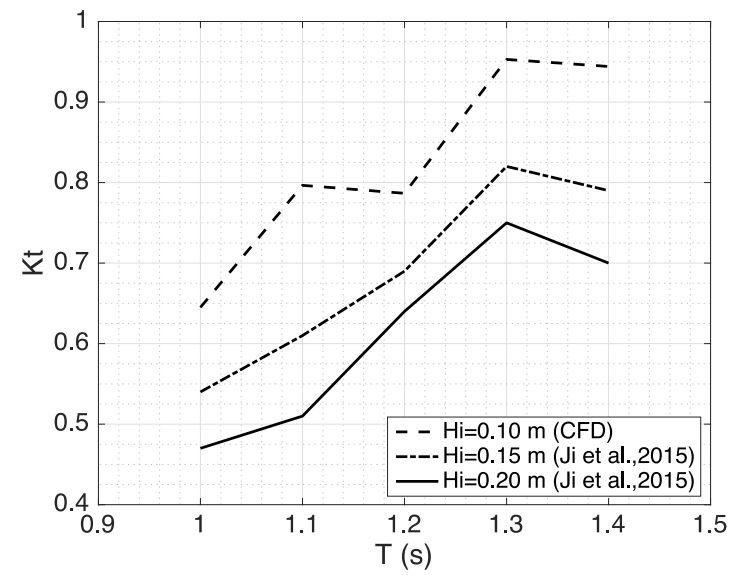

Figure 7. Transmission coefficients for various incident wave heights 
Figure 7 shows further CFD prediction on transmission coefficient for $0.1 \mathrm{~m}$ incident wave height with various wave periods in which associated with environmental parameters of South China Sea. The results seem realistic by showing an increasing attribute on wave transmissions in relation to experimental measurements. Since this wave height is more susceptible to the variable trend of transmission coefficients, the optimization problem would be based on the referred wave parameter including various wavelengths. This validation is important for subsequent study on the optimization of lateral separation between twin pontoons of floating breakwater.

\section{Optimization Results}

Elitist non-dominated sort genetic algorithm (NSGA-II) is run with a population size of 100 and for 100 generations. The variables are used as real numbers with GA parameters of a simulated binary crossover $(\mathrm{SBX})$ recombination operator with $p_{c}=0.9$ and distribution index $\eta_{c}=10$ and a polynomial mutation operator with $p_{m}=0.5$ and distribution index of $\eta_{m}=20$. Figure 8 to 11 present the entire competing trade-offs, projecting all elite solutions evaluated in a two-dimensional space between the design objectives specified: minimization of the wave transmission (objective 1) and maximize energy dissipation (objective 2). The problem has a convex solution of global Pareto-optimal front.

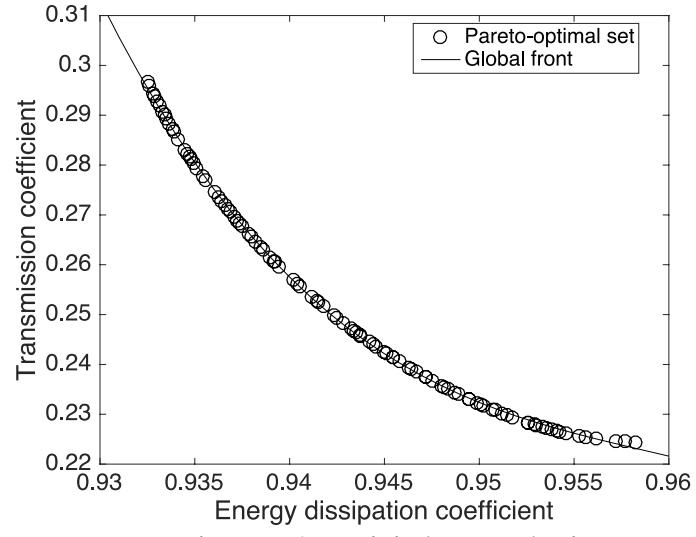

Figure 8. Initial population.

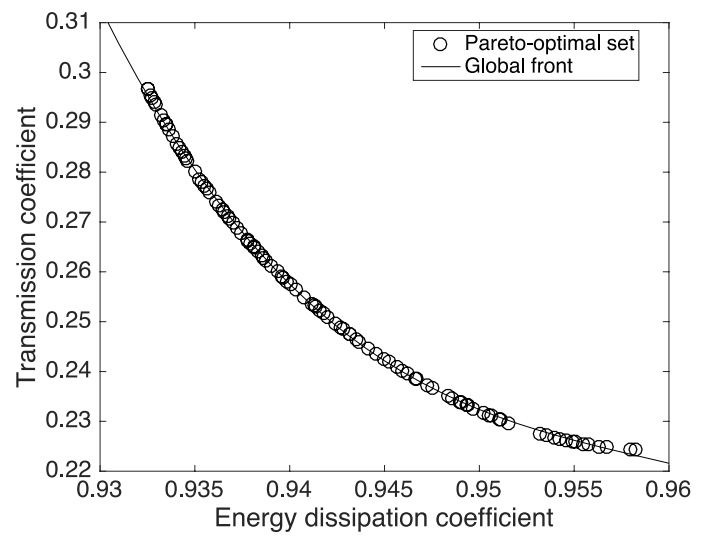

Figure 10. Population at generation 100.

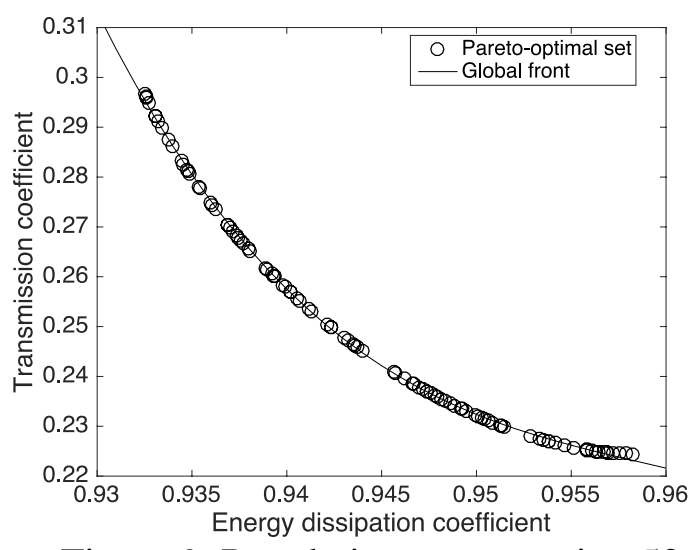

Figure 9. Population at generation 50.

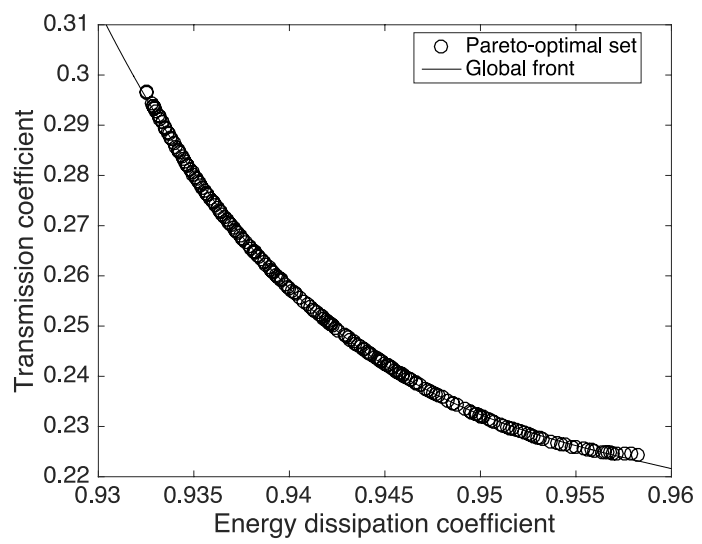

Figure 11. Population at generation 500. 
Figure 8 is the initial population shown on the objective space. Figures 9 and 10 show populations at generations 50 and 100 respectively. The figures illustrate how the genetic operators cause the population to move and spread over the global front with generations. At initial generation, the population converges very well to the true front but diverse poorly among non-dominated solutions. At generation 50 and 100, a better spread of the solutions is obtained however the diversity towards extreme solutions does not sorting very well to the global front.

By increasing iteratively, the number of generation and population up to 500 and 200 respectively, Figure 11 depicts the finalized Pareto-optimal set associated with no further change in plot movement. At this generation, the population comes very close to the global Pareto-optimal front while maintaining diversity among obtained solutions. Here, the convergence metric, $Y$ and diversity metric, $\Delta$ are approximately 0.000102 and 0.01598 respectively. The smaller ratios of these performance measures indicate good convergence and spread between solutions.

From Figure 8 to 11, it is clear that all non-dominated solutions consistently comprise a true Pareto front and thus a globally optimal trade-off is found in this research. Referring to an extreme solution in Figure 11, the optimum S/D ratio results to 2.72. At this lateral separation, the wave attenuation of 0.225 will be expected to achieve by the floating breakwater.

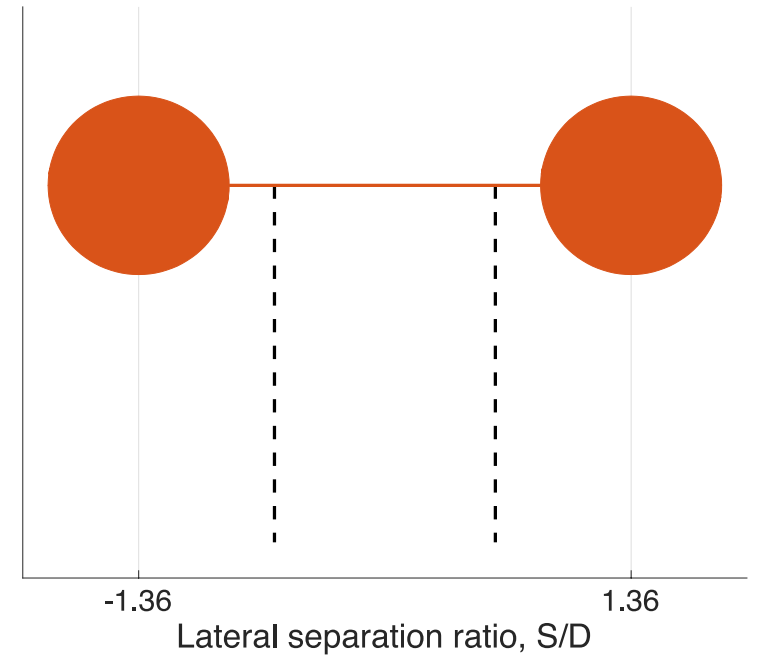

Figure 12. Optimum lateral separation of TPFB 
(a)

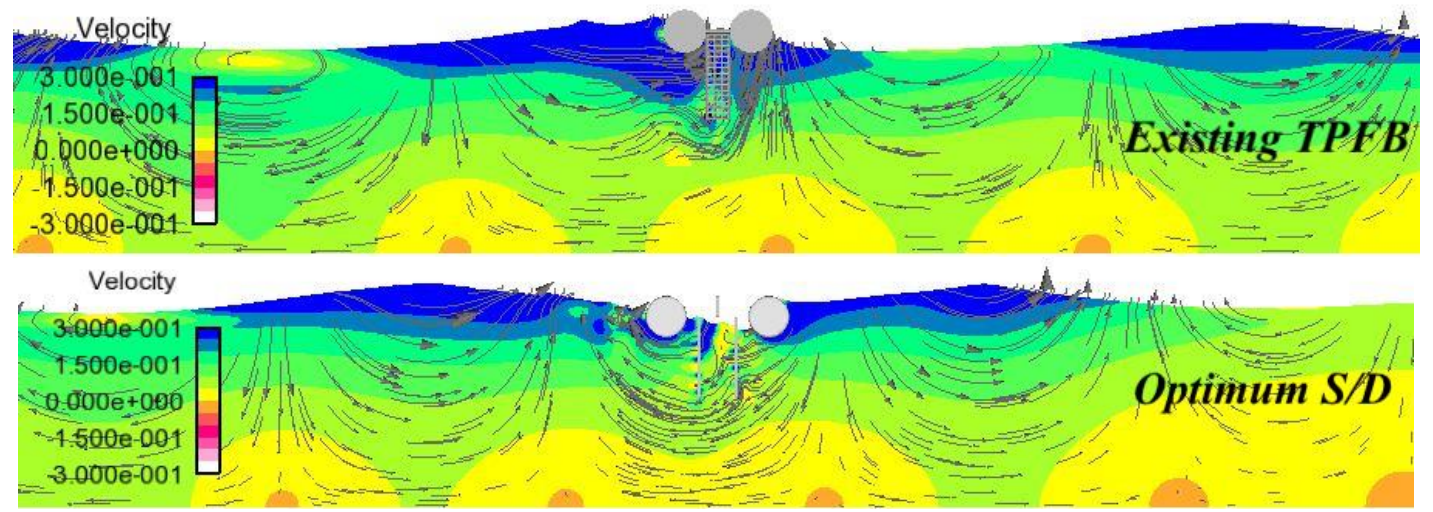

(b)
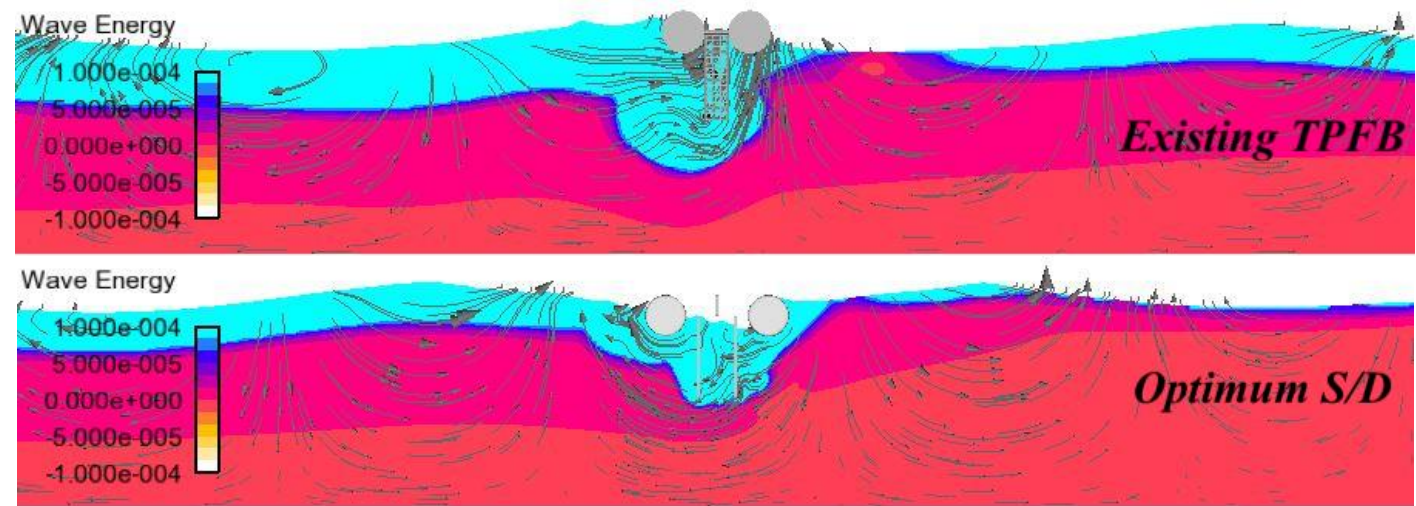

Figure 13. Wave absorbing effects of existing and optimum S/D of TPFB in a characteristic of (a) velocity pattern (b) wave energy pattern.

Figure 13 (a) and (b) depict the visualization on the reduction of flow parameters observed in CFD simulation for optimum S/D when compared to existing model at $H=0.15 \mathrm{~m}$ and $T$ $=1.4 \mathrm{~s}$. Referring to the flow pattern, it is clear that the velocity fields before and after the floating breakwater markedly decreases as the S/D ratio moves to optimum one which can be traced by the blue-green scale color. Here, significant reductions on the velocity magnitudes at front and leeside of TPFB with optimum S/D lead to proportional reduction in wave energy compared to existing TPFB counterpart. Hydrodynamically, a drop in this leeward wave energy directly reduces wave crest and thus wave transmission, $K_{t}$. Results from CFD simulation justify the consequence is caused by the dispersing effect of wave energy particularly due to the in-plane stiffness damping induced by the net cage (Abdullah et al. (2017)). In short, the results demonstrate the efficiency of GA search to determine an optimum S/D that proportionally results to optimum hydrodynamic performance of TPFB. 


\section{CONCLUSION}

The numerical optimization modelling on the lateral separation, S/D between twin pontoons of floating breakwater was carried out in this research using genetic algorithm (GA). Various S/D ratios and wavelengths were analyzed using CFD approach prior to optimization process. The optimization results showed that using a planned and proper strategies in defining the problem, the obtained GA solutions obviously do not suffer from poor convergence even at an early generation. The result investigation in two-dimensional space reveals the robustness of the current GA computation that can search a globally optimal trade-off between design objectives. As compared to existing model of TPFB, $K_{t}$ minimized to less than 0.3 whereas $K_{d}$ maximized to greater than 0.95 resulting to optimum S/D indicated by significant reduction in flow parameters observed at leeward area of the floating breakwater. From engineering view, the concept of present optimization is the key for better wave attenuation by floating breakwaters especially in coastal waters, for instance to particularly avoid from insufficient wave damping and strong reflection which proportionally result to sediment erosion both to protected and adjacent areas.

\section{ACKNOWLEDGEMENTS}

The authors would like to thank as well a great appreciation to the Universiti Malaysia Terengganu for its support upon the completion of this research.

\section{REFERENCES}

[1] Fitriadhy A, Faiz MA, Abdullah SF. Computational fluid dynamics analysis of cylindrical floating breakwater towards reduction of sediment transport. Journal of Mechanical Engineering and Sciences. 2017;11:3072-3085.

[2] McCartney. Floating breakwater design. Journal of Waterway, Port, Coastal, and Ocean Engineering. 1985;111:304-318.

[3] He F, Huang ZH, Adrian WKL. An experimental study of floating breakwater with asymmetric chambers for wave energy extraction. Applied Energy. 2013;106:222231.

[4] Moghim MN, Botshekan M. Analysis of the performance of pontoon-type floating breakwaters. HKIE Transactions. 2017;24:9-16.

[5] Mani JS. Design of Y-frame floating breakwater. Journal of Waterway, Port, Coastal, and Ocean Engineering. 2014;117:105-119.

[6] Erik DC, Harry BB, Andreas PSF, Alexander KL, Karsten LJ. An experimental and numerical study of floating breakwater. Coastal Engineering. 2018;137:43-58.

[7] Murali K, Mani JS. Performance of cage floating breakwater. Journal of Waterway, Port, Coastal, and Ocean Engineering. 1997;123:172-179.

[8] Murali K, Amer SS, Mani JS. Dynamics of cage floating breakwater. Journal of Offshore Mechanics and Arctic Engineering. 2005;127:331.

[9] Ji C-Y, Chen X, Cui J, Yuan Z-M, Incecik A. Experimental study of a new type of floating breakwater. Ocean Engineering. 2015;105:295-303. 
[10] Ji C-Y, Yu-Chan GUO, Cu J, Yuan Z-M, Ma X-J. 3D experimental study on a cylindrical floating breakwater system. Ocean Engineering. 2016;125:38-50.

[11] Ji C-Y, Cheng Y, Yang K, Oleg G. Numerical and experimental investigation of hydrodynamic performance of a cylindrical dual pontoon-net floating breakwater. Coastal Engineering. 2017;129:1-16.

[12] Brebner A, Ofuya AO. Floating breakwaters. In Proceeding of the $11^{\text {th }}$ Conference on Coastal Engineering, ASCE. 1968;2:1055-1094.

[13] Williams AN, Abul-Azm AG. Dual pontoon floating breakwater. Ocean Engineering. 1997;24:465-478.

[14] Syed SA, Mani JS. Performance of rigidly interconnected multiple floating pontoons. Journal of Naval Architecture and Marine Engineering. 2004;1:3-17.

[15] Abdullah SF, Fitriadhy A, Hairil M, Jusoh A. Hydrodynamic performance of cylindrical floating breakwater in waves. International Journal of Automotive and Mechanical Engineering. 2017;14:4715-4729.

[16] Negnevitsky M. Artificial intelligence: A guide to intelligent systems: Pearson education, 2005.

[17] Hendricks D, Gebbie T, Wilcox D. High-speed detection of emergent market clustering via an unsupervised parallel genetic algorithm. South African Journal of Science. 2016;112:01-09.

[18] Castillo O, Melin P, Kacprzyk J. Soft computing for hybrid intelligent system. Berlin, Germany: Springer, 2008.

[19] Deb K, Pratap A, Agarwal S, Meyarivan T. A fast elitist multiobjective genetic algorithm: NSGA-II. IEEE Transactions on Evolutionary Computation. 2002;6:182197.

[20] Beyer H-G, Deb K. On self-adaptive features in real-parameter evolutionary algorithm. IEEE Trabsactions on Evolutionary Computation. 2001;5:250-270.

[21] Deb K, Agarwal RB. Simulated binary crossover for continuous search space. Complex Systems. 1995;9:115-148.

[22] Peng W, Lee K-H, Shin S-H, Mizutani N. Numerical simulation of interactions between water waves and inclined-moored submerged floating breakwaters. Coastal Engineering. 2013;82:76-87.

[23] Flow3D 10.1.1 User Manual: Flow Science Inc., 2013.

[24] Koutsourakis N, Bartzis JG, Markatos NC. Evaluation of Reynolds stress, k- $\varepsilon$ and RNG $\mathrm{k}-\varepsilon$ turbulence models in street canyon flows using various experimental datasets. Environmental Fluid Mechanics. 2012:1-25.

[25] Goda Y, Suzuki Y. Estimation of incident and reflected waves in random wave experiments. In Proceedings of the $15^{\text {th }}$ International Conference on Coastal Engineering, ASCE. 1976:828-845. 